\title{
Use of ICG videoangiography and FLOW 800 analysis to identify the patient-specific venous circulation and predict the effect of venous sacrifice: a retrospective study of 172 patients
}

\author{
Francesco Acerbi, MD, PhD, Ignazio G. Vetrano, MD, Tommaso Sattin, MD, Jacopo Falco, MS, \\ Camilla de Laurentis, MD, Costanza M. Zattra, MD, Lorenzo Bosio, MD, Zefferino Rossini, MD, \\ Morgan Broggi, MD, PhD, Marco Schiariti, MD, and Paolo Ferroli, MD
}

\begin{abstract}
Neurosurgical Unit II, Department of Neurosurgery, Fondazione IRCCS “Istituto Neurologico C. Besta," Milan, Italy
OBJECTIVE The best management of veins encountered during the neurosurgical approach is still a matter of debate. Even if venous sacrifice were to lead to devastating consequences, under certain circumstances, it might prove to be desirable, enlarging the surgical field or increasing the extent of resection in tumor surgery. In this study, the authors present a large series of patients with vascular or oncological entities, in which they used indocyanine green videoangiography (ICG-VA) with FLOW 800 analysis to study the patient-specific venous flow characteristics and the management workflow in cases in which a venous sacrifice was necessary.
\end{abstract}

METHODS Between May 2011 and December 2017, 1972 patients were admitted to the authors' division for tumor and/ or neurovascular surgery. They retrospectively reviewed all cases in which ICG-VA and FLOW 800 were used intraoperatively with a specific target in the venous angiographic phase or for the management of venous sacrifice, and whose surgical videos and FLOW 800 analysis were available.

RESULTS A total of 296 ICG-VA and FLOW 800 studies were performed intraoperatively. In all cases, the venous structures were clearly identifiable and were described according to the flow direction and speed. The authors therefore defined different patterns of presentation: arterialized veins, thrombosed veins, fast-draining veins with anterograde flow, slow-draining veins with anterograde flow, and slow-draining veins with retrograde flow. In 16 cases we also performed a temporary clipping test to predict the effect of the venous sacrifice by the identification of potential collateral circulation.

CONCLUSIONS ICG-VA and FLOW 800 analysis can provide complete and real-time intraoperative information regarding patient-specific venous drainage pattern and can guide the decision-making process regarding venous sacrifice, with a possible impact on reduction of surgical complications.

https://thejns.org/doi/abs/10.3171/2018.4.FOCUS18120

KEYWORDS cerebral veins; clipping test; FLOW 800; ICG; indocyanine green; software analysis; venous sacrifice; videoangiography

$\mathrm{T}$ HE best management of veins encountered during a neurosurgical procedure is still a matter of debate. In particular, because venous sacrifice can lead to devastating consequences, ${ }^{3,19-21,23-25,28,31,33-35}$ the elective sacrifice of an important vein is usually considered unsafe. However, venous sacrifice may actually prove to be desirable, under certain circumstances, to enlarge the surgical exposure of the targeted site and also to increase the extent of the resection in tumor surgery. Nonetheless, there are few studies that systematically explore the effect of venous sacrifice in neurosurgery. ${ }^{13,14,19,20,30,35}$ Most often, neurosurgeons base their decision of whether or not to sacrifice a vein on their own surgical experience and on anatomical location of the vein rather than on evidencebased data. ${ }^{8}$ In a retrospective series of patients operated on in the period 2006-2009, our group evaluated the use of indocyanine green videoangiography (ICG-VA) to study intraoperatively the different characteristics of ve-

ABBREVIATIONS AVF = arteriovenous fistula; $\mathrm{AVM}=$ arteriovenous malformation; $\mathrm{GBM}=$ glioblastoma; $\mathrm{ICG}-\mathrm{VA}=$ indocyanine green videoangiography; $\mathrm{MCA}=$ middle cerebral artery.

ACCOMPANYING EDITORIAL DOI: 10.3171/2018.4.FOCUS18207.

SUBMITTED March 1, 2018. ACCEPTED April 6, 2018.

INCLUDE WHEN CITING DOI: 10.3171/2018.4.FOCUS18120. 
nous flow, not only in pathological veins but also in normal veins, and we proposed the use of an ICG temporary clipping test to predict the effect of venous sacrifice. ${ }^{13,14,32}$ Since then, the evolution of the ICG-VA, through the integration of a new software into the surgical microscope (FLOW 800, Carl Zeiss Meditec), has allowed the performance of a semiquantitative flow analysis that has improved our understanding of the pathophysiology of arterial, venous, and parenchymal blood flow in neurosurgical procedures. ${ }^{4,7,22}$

The purpose of the present study was to evaluate, in a large series of patients with vascular and oncological diseases, the venous flow characteristics and the management workflow in cases in which a venous sacrifice was needed based on the use of ICG-VA with FLOW 800 analysis.

\section{Methods \\ Patients}

From May 2011 to December 2017, 1972 patients underwent a neurosurgical procedure for tumor or vascular disease at Neurosurgical Unit II of the Fondazione IRCCS "Istituto Neurologico C. Besta." We retrospectively reviewed all cases in which ICG-VA with FLOW 800 analysis was used intraoperatively with a specific target in the venous angiographic phase or for the management of venous sacrifice, and whose surgical videos and FLOW 800 analysis were available. Based on these criteria, we found 126 vascular procedures (48 intracranial aneurysms, 25 revascularization procedures, 16 arteriovenous malformations [AVMs], 14 cranial and spinal arteriovenous fistulas [AVFs], 8 cavernous hemangiomas, and 15 microvascular decompressions) and 46 tumor surgeries (7 spinal cord tumors [5 ependymomas, 1 pilocytic astrocytoma, and 1 enterogenous cyst], 12 meningiomas, 9 glioblastomas [GBMs], 1 anaplastic oligodendroglioma, 5 tumors of the pineal region, 3 hemangioblastomas, 3 metastatic tumors, 2 central neurocytomas, 1 lumbar intradural neuroendocrine tumor, 1 cervical intradural schwannoma, 1 pleomorphic xanthoastrocytoma, and 1 decompressive hemicraniectomy for edema and raised intracranial pressure after resection of multiple atypical meningiomas); in these cases, ICG-VA was performed with the specific aim of evaluating venous flow, for a total of 296 videoangiographic studies.

All patients gave their informed consent with regard to undergoing ICG-VA. The local ethical committee approved the database that was used to perform the retrospective analysis.

\section{Venous ICG-VA With FLOW 800 Analysis}

ICG-VA was performed as previously described, ${ }^{1,11,13}$ with intravenous ICG administration of a standard dose of $12.5 \mathrm{mg}$ in a single bolus, each time it was necessary, never exceeding the maximum daily dose of $5 \mathrm{mg} / \mathrm{kg}$. The flow analysis was performed using the FLOW 800 software, integrated in the surgical microscope (Fig. 1). ${ }^{22}$

The effect of venous sacrifice, when performed, was assessed specifically by ICG-VA with FLOW 800 analysis. Furthermore, for cases in which venous sacrifice of bridging veins was considered, either to enlarge the surgi- cal corridor or to achieve a more radical tumor resection, a temporary occlusion test, under ICG and FLOW 800 analysis guidance, was performed, as previously described. ${ }^{11-13}$ Briefly, a temporary clip (or, more simply, the closed tips of bipolar forceps) was placed on the vein to be tested and flow direction and dynamics were analyzed by using ICGVA and FLOW 800 (the so-called ICG temporary clipping test). Flow stagnation was considered to indicate a lack of collateral circulation, whereas flow reversal was interpreted as a sign of the presence of collateral circulation.

\section{Results}

\section{Assessment of Venous Flow Characteristics by ICG-VA With FLOW 800 Analysis}

Venous flow could be visualized and characterized based on the ICG-VA and FLOW 800 algorithm, with excellent image quality, in all cases. No adverse reaction to ICG administration was registered in our series.

We could differentiate the venous flow in 5 different patterns.

\section{Arterialized Veins}

The presence of an AVF causes venous arterialization with a flow that has similar characteristics to the arterial one. These veins are easily recognizable, even without ICG-VA, because of their red color. However, the FLOW 800 analysis was particularly useful to assess the arterialization of one or more veins exposed in the surgical approach, both on the "delay map" and on the specific flow curves in normal and arterialized veins, reconstructed by the software. This pattern of venous flow could be found in 16/16 AVMs, 14/14 AVFs, and 11 tumors (3 GBMs, 3 hemangioblastomas, 1 tumor of the pineal region, 1 lumbar intradural neuroendocrine tumor, 1 cervical intradural schwannoma, 1 pleomorphic xanthoastrocytoma, and 1 metastasis) (Fig. 1).

\section{Thrombosed Veins}

A portion of a vein was observed to be thrombosed at the start of the surgical procedure. It was observed in a case of left temporal GBM in which the tumor encased the vein of Labbé. A residual portion of the tumor was initially left around the vein because it was impossible to dissect it from the venous wall. ICG-VA was performed to specifically assess the venous flow, and it showed thrombosis of a portion of the Labbé vein encased by the tumor, with a retrograde flow of a basal temporal vein toward the superficial sylvian vein (Fig. 2).

\section{Fast Bridging Veins With Anterograde Flow Toward the Sinuses}

These veins exhibited fast and homogeneous filling, with an anterograde flow toward the usual draining sinus, and a time to half-maximal fluorescence that was within 5 seconds of the arterial phase, as evident in the delay maps reconstructed by the FLOW 800 algorithm. We identified this pattern of venous flow in parasagittal veins, sylvian veins, and infratentorial veins (both supracerebellar and superior petrosal veins) (Fig. 3, Video 1).

VIDEO 1. A temporary clipping test performed on a fast-draining superficial sylvian vein draining anterogradely to the sphenoparietal 

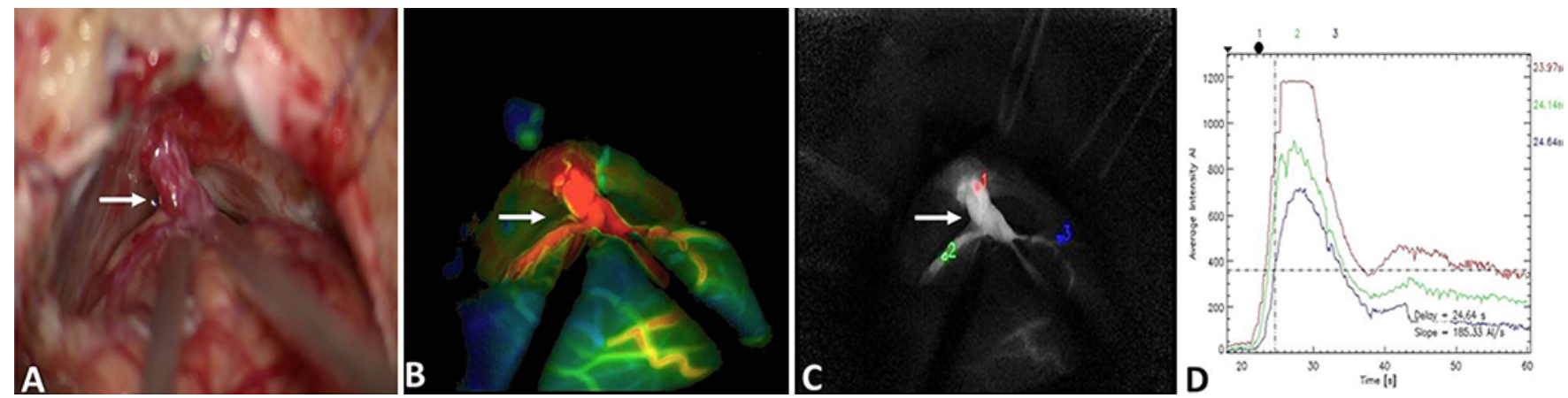

FIG. 1. ICG-VA reconstructs the map of fluorescence intensity on the basis of average intensity units (AI); it is also possible to build a color map of the half-maximal fluorescence, corresponding to the delay map. Moreover, a manual selection of different regions of interest (ROIs) up to 8 in the map of gray intensity can determine a flow curve corresponding to each ROI, indicated with different colors and numbers. A: A right retrosigmoid approach for tentorial AVF in the region of the superior petrosal sinus, with retrograde cortical drainage in Dandy's veins (arrow). B: The delay map obtained with FLOW 800 analysis showed the red arterialized venous flow. $\mathbf{C}$ and $\mathbf{D}$ : The selected ROls from 1 to 3 generated a diagram with the different flow curves, which confirmed the arterialized and retrograde flow, with a slower time to half-maximal fluorescence in ROIs 2 and 3, compared with ROI 1.

sinus for the surgical approach to an anterior communicating artery aneurysm. A clip was positioned in the proximal part of the vein, closer to the sphenoparietal sinus, and ICG-VA identified flow stagnation in the distal part of the vein, which was promptly resolved by clip removal. The ICG clipping test was considered negative, and the vein was not sacrificed. After clipping of the aneurysm, ICG-VA was repeated to confirm aneurysm exclusion, and it also confirmed vein patency (red arrow). Copyright Fondazione IRCCS "Istituto Neurologico C. Besta." Published with permission. Click here to view.

\section{Slow Bridging Veins With Anterograde Flow Toward Sinuses}

These veins exhibited a slow and nonuniform filling, usually with multiple tributaries and one large collector close to the sinus and with multiple streams with different flow velocities that are easily recognizable on the delay maps and that could be better clarified by the flow curves performed with the FLOW 800 algorithm. This venous flow pattern was found more commonly in parasagittal and sylvian veins and only rarely in supracerebellar and superior petrosal veins (Fig. 4, Video 2).

VIDEO 2. A temporary clipping test performed on a slow-draining parasagittal bridging vein, draining anterogradely to the superior sagittal sinus (SSS) for the surgical approach to a pericallosal aneurysm. An ICG-VA with FLOW 800 analysis was performed after dural opening and vein dissection to assess venous flow characteristics. Then a clip was positioned on the main collector close to the SSS, and ICG-VA with FLOW 800 analysis was again performed to confirm the presence of good collateral circulation. The ICG temporary clipping test was considered positive and the vein was sacrificed. After clipping of the aneurysm, ICG-VA was repeated to confirm aneurysm exclusion, and it also confirmed the persistence of good collateral flow. Copyright Fondazione IRCCS "Istituto Neurologico C. Besta." Published with permission. Click here to view.

\section{Slow Bridging Veins With Retrograde Flow Opposite the Sinuses}

This pattern was found in two veins intimately related to parasagittal meningiomas, after meningioma removal. ICG-VA with FLOW 800 analysis was performed to assess the patency and flow characteristics of the vein after meningioma removal. In both cases, the flow was slow and retrograde, in a direction opposite the sinus, as shown in the delay map and flow curve analysis (Fig. 5).

\section{Management of Venous Sacrifice Based on ICG-VA With FLOW 800 Analysis}

Management of Arterialized Veins in AVMs, AVFs, and Tumors

Our attitude in cases of arterialized veins was to disconnect the AVF from the main venous collector that was arterialized in the presurgical survey, with the aim of restoring the normal physiological venous flow. A check with ICG-VA and FLOW 800 analysis was always performed in vascular cases and in selected tumor cases (5 of 11 cases with vein arterialization) at the end of the surgical procedure. This study confirmed the normalization of venous flow in the previous arterialized vein, in all
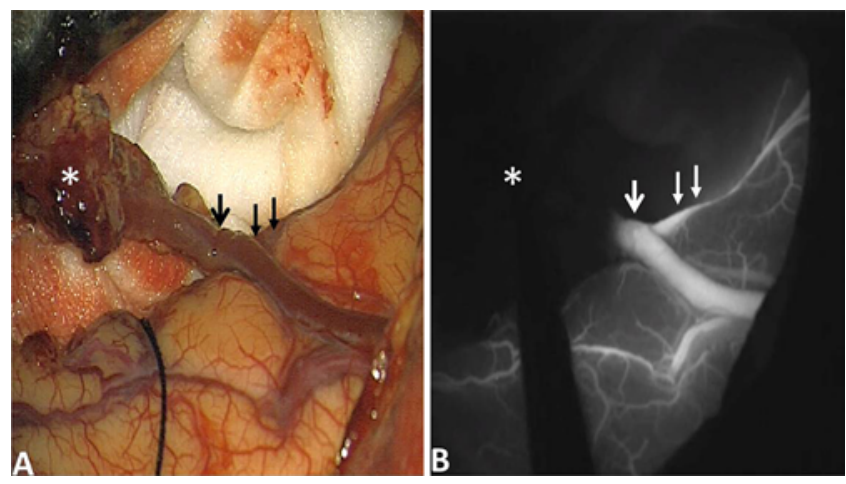

FIG. 2. A: After resection of a left temporal GBM, the left vein of Labbé was found to be encased by residual neoplastic tissues (asterisk), which was considered to be unsafe for dissection from the vein wall. B: ICGVA showed that the proximal part of the vein closer to the transverse sinus was already thrombosed (asterisk), with a collateral circulation that had developed in the distal part of the vein, draining toward the sylvian venous system (black arrows in A, white arrows in B). Therefore, it was possible to sacrifice the vein of Labbé without adverse consequences, removing the tumor completely. 

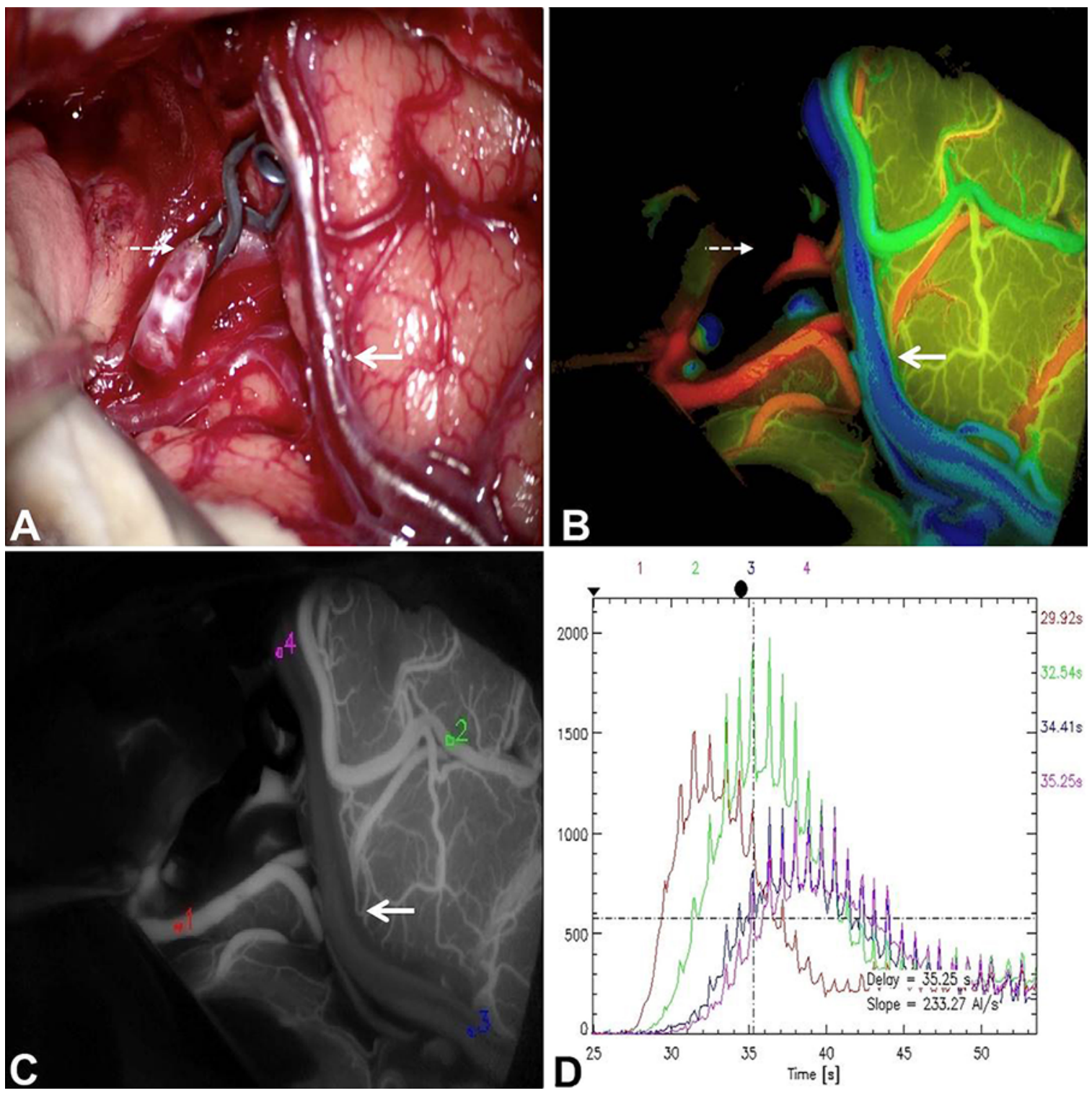

FIG. 3. A: Intraoperative photograph of a right MCA aneurysm that was surgically clipped (dashed arrow) through a pterional approach. B: ICG-VA was performed to confirm a complete aneurysm exclusion (dashed arrow). The color delay map, reconstructed based on time to half-maximal fluorescence, also showed the flow in the superficial sylvian vein located on the temporal side of the sylvian fissure. The vein (white arrow in B) was found to have a homogeneous flow toward the sphenoparietal sinus. C and D: In addition, the analysis of flow curves, in frontal (1) and temporal (2) arteries and in the superficial sylvian vein (3 and 4), allowed us to measure a minimal arteriovenous difference in time to half-maximal fluorescence of 4.49 seconds (range 34.41-29.92 seconds), thus confirming the fast flow (within 5 seconds of arterial flow) and the anterograde flow direction (based on the faster time to halfmaximal fluorescein in ROI 3 compared with ROI 4).

tumor cases, in 10 AVM cases, and in 10 AVF cases (Fig. 6A-H). In 6 AVM and 4 AVF cases, an arterialized vein was shown to be dark or receiving only retrograde flow from normal continuous veins at the end of the surgical procedure, due to the fact that the vein was not important for the normal physiological venous drainage.

Evaluation of the Effect of Venous Sacrifice Without ICG Clipping Test

In 10 cases, ICG-VA was performed at the end of the surgical procedure to assess the effect of unintended or obliged venous sacrifice in veins not studied by ICG clipping test.

In 7 middle cerebral artery (MCA) aneurysms, one or more bridging veins in the sylvian fissure, connecting the frontal and temporal lobes, were sacrificed to enlarge the surgical corridor to the aneurysms, never damaging the main superficial sylvian trunk. In these cases, the ICG-VA, performed at the end of the surgical procedure to check the effect of aneurysm clipping, was also used to confirm a good venous outflow in the frontal and temporal lobes.

In 3 meningiomas (2 parasagittal and 1 sphenoorbital), 

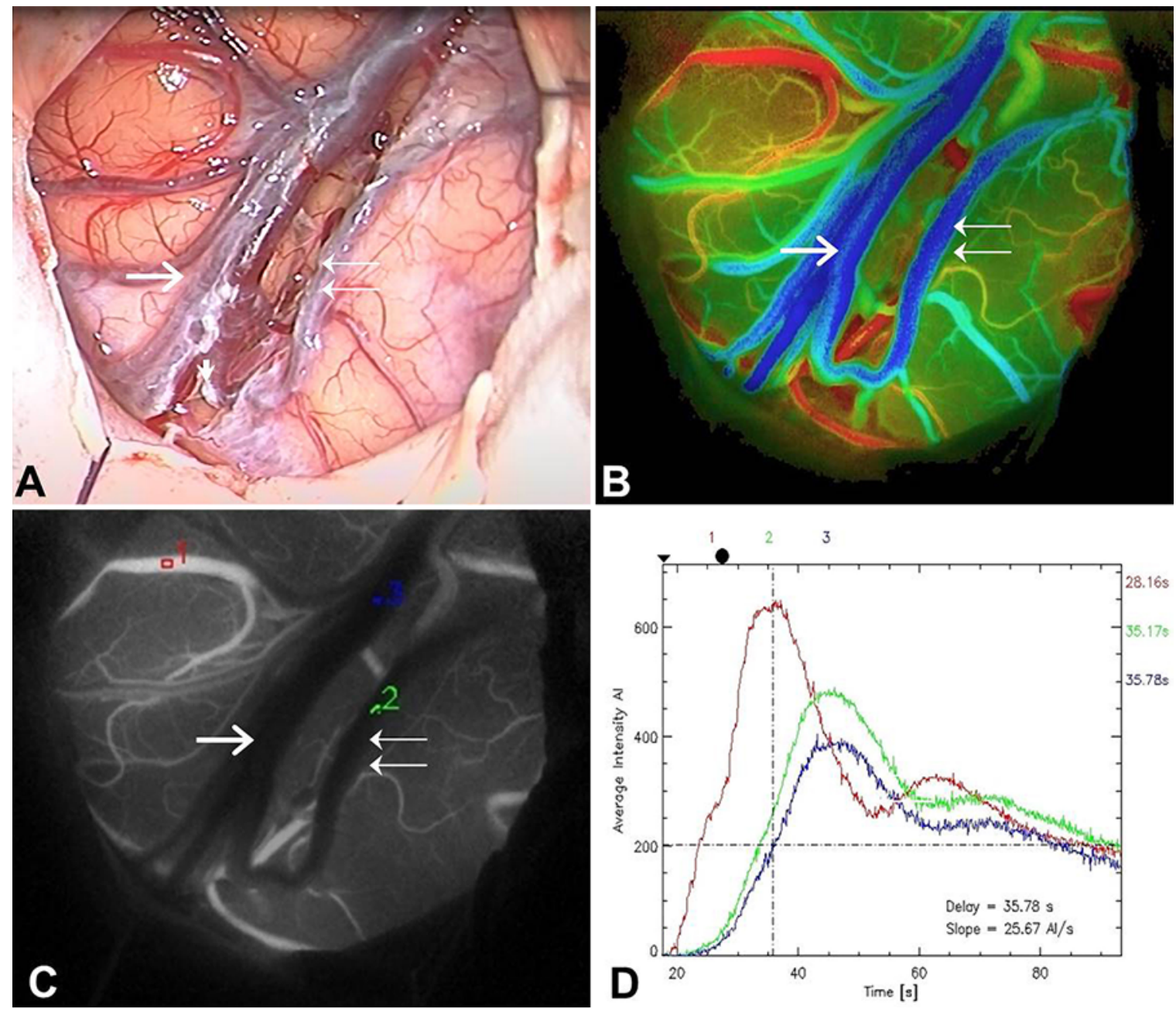

FIG. 4. A: A case of sylvian fissure opening for the removal of a left insular glioma. B-D: ICG-VA and FLOW 800 analysis were performed to study the venous flow characteristics of the superficial sylvian vein system. The color delay map, based on time to half-maximal fluorescence $(B)$, showed the presence of a nonuniform filling in the veins, with multiple streams inside the different compartments (dark blue, light blue, and green in B) and multiple tributaries to the larger superficial sylvian vein. The flow curve analysis (C and D), based on 3 ROls positioned in a temporal artery (1) and in two venous components (2 and 3), confirmed a slow-draining venous flow with a minimal arteriovenous difference in time to half-maximal fluorescence of 7.01 seconds and also different flow velocities in the two veins (double arrows and single arrow in C, green and blue curves in D, time to half-maximal fluorescence of 35.17 and 35.78 seconds, respectively).

a venous sacrifice on bridging veins was deemed necessary to completely remove the tumor that was encasing the more proximal venous trunk, closer to the superior sagittal or sphenoparietal sinuses. In this case, ICG-VA at the end of the surgical procedure was used to confirm the presence of good collateral circulation, in a direction opposite the usual outflow toward the sinuses (in 2 cases the superior sagittal sinus and in 1 case the sphenoparietal sinus).

In one case of GBM described in a previous section, ICG-VA was used to verify the flow in one vein of Labbé completely encased by residual tissue (Fig. 2A); this study showed the thrombosis of the more proximal part of the vein of Labbé, with the distal part functioning as a connection of one basal temporal vein to drain toward the superficial sylvian system (Fig. 2B).
Management of Venous Sacrifice by ICG Temporary Clipping Test

An ICG temporary clipping test was performed in 16 cases (7 vascular and 9 tumor cases) on different bridging veins, draining to the superior sagittal, sphenoparietal, and superior petrosal sinuses; to the tentorium; and in one posterior median sulcus vein in the spinal cord.

The clipping test was performed to establish the necessity of a venous sacrifice to enlarge the surgical corridor in 7 vascular cases, while in 9 tumors, it was performed to determine the necessity of venous sacrifice to enlarge the surgical corridor to the lesion or to completely remove the tumor.

In 5 cases $(1$ pericallosal aneurysm, 1 intraventricular neurocytoma, 1 frontal metastasis, 1 frontal GBM, and 1 meningioma), the test was performed on slow-draining 

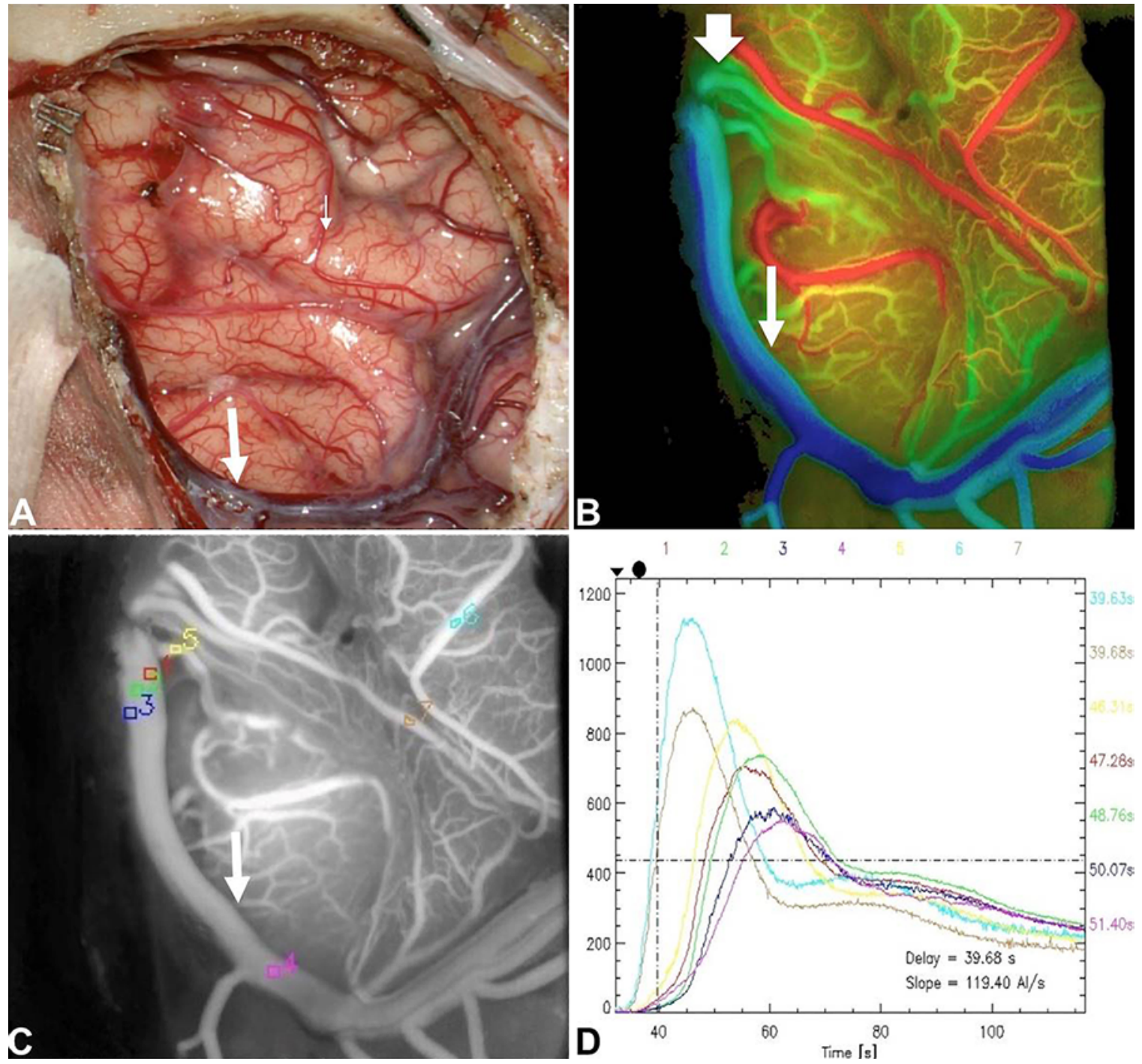

FIG. 5. A case of parasagittal meningioma after resection. A: A draining vein to the superior sagittal sinus, intimately involving the meningioma, was dissected and preserved during surgery (large white arrow). ICG-VA and FLOW 800 analysis were performed only at the end of tumor resection to assess the patency and flow characteristics of the vein. B: The color delay map, based on time to half-maximal fluorescence, showed a nonuniform flow with multiple streams in the cortical draining vein (dark blue, light blue, and cyan color, thin white arrow) receiving multiple smaller and faster veins from the frontal parenchyma (green color and thick arrow) and having a retrograde flow, opposite the superior sagittal sinus. C and D: Flow curve analysis confirmed the retrograde and extremely slow flow (faster time to half-maximal fluorescence in ROI 5 compared with ROIs 2, 1, 3, and 4; minimal arteriovenous difference varying from 7.65 to 11.77 seconds based on the different ROls).

parasagittal veins draining anterogradely to the superior sagittal sinus that were obstructing the surgical corridor (the first 4 cases) or that were encased by the tumor (the last case of parasagittal meningioma). In 4 cases, the ICG temporary clipping test was positive, with good collateral circulation after clipping, and the respective vein was sacrificed without consequences (Fig. 7, Video 2). In one case, the collateral retrograde flow was considered too slow (3 seconds of difference compared with other veins) and the vein was draining an eloquent area (primary motor cortex), and thus it was considered not suitable for sacrifice.

In 6 cases ( $1 \mathrm{MCA}$ aneurysm, 2 uncal cavernomas approached via a transsylvian approach, 2 cases of moya- moya disease submitted to superior temporal artery-MCA bypass, and 1 insular left glioma), the test was performed on slow-draining sylvian veins with anterograde flow to the sphenoparietal sinus that were obstructing the corridor to the surgical target. In all 6 cases, the ICG temporary clipping test was positive, with good collateral circulation after clipping, and the respective vein was sacrificed without consequences.

In 1 case (1 anterior communicating artery aneurysm), the test was performed on a fast-draining sylvian vein with anterograde drainage to the sphenoparietal sinus, which was limiting the opening of the sylvian fissure to enlarge the surgical corridor to the aneurysm. The ICG temporary 

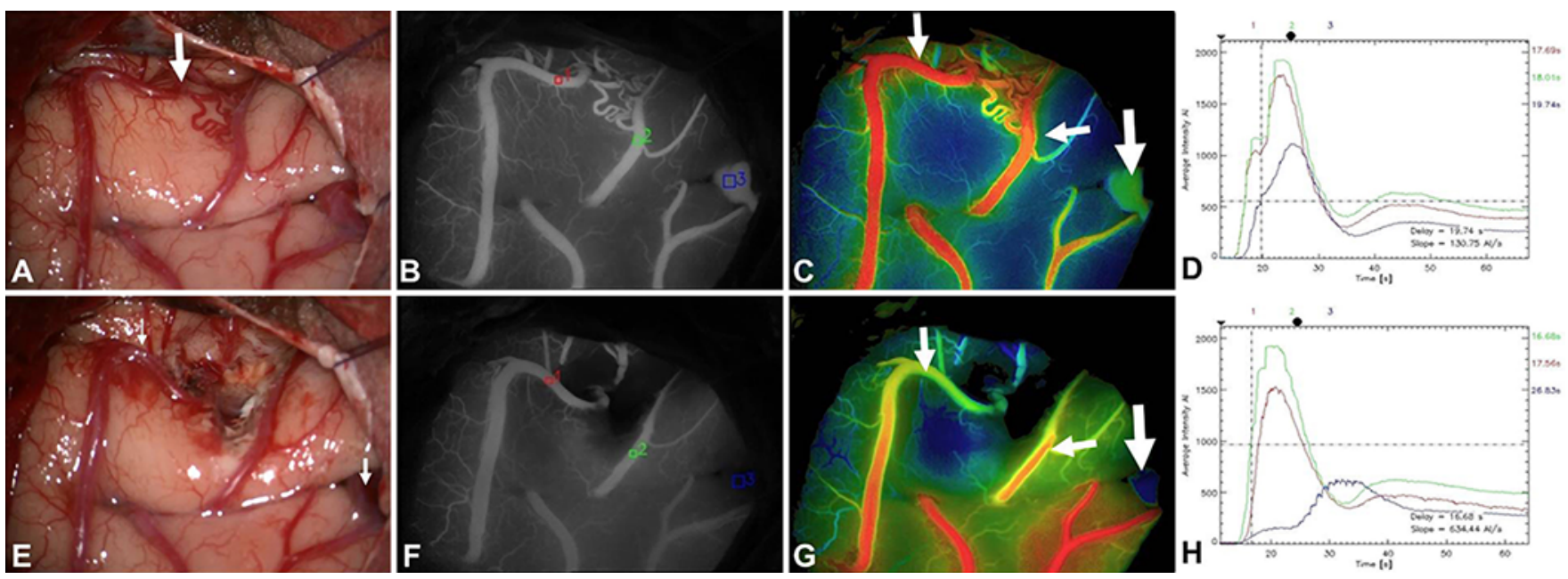

FIG. 6. Management of arterialized veins in a case of Spetzler-Martin grade I right temporobasal AVM. Before AVM resection (arrow, A), an ICG-VA with FLOW 800 analysis (B-D) showed two feeders (ROls 1 and 2 in B, thin arrows in C) and an arterialized draining vein (ROI 3 in $\mathrm{B}$, thick arrow in $\mathrm{C}$ ). The flow curve analysis better clarified the vein arterialization with an arteriovenous difference of 2.05 seconds. E-H: The same analysis was performed after AVM resection (E), confirming arterial and venous patencies (ROIs 1-3 in F, thin arrows and thick arrow in G). Furthermore, the delay map and flow curve analysis (H) showed venous flow normalization, with an arteriovenous difference of 10.15 seconds.

clipping test was negative, with flow stagnation in the vein that was therefore not considered suitable for sacrifice (Video 1).

In 3 cases ( 2 tumors of the pineal region and 1 menin- gioma of the petrous apex), the test was performed on posterior fossa veins (2 slow-draining supracerebellar veins and 1 fast-draining superior petrosal vein).

In the fast-draining superior petrosal vein, the ICG
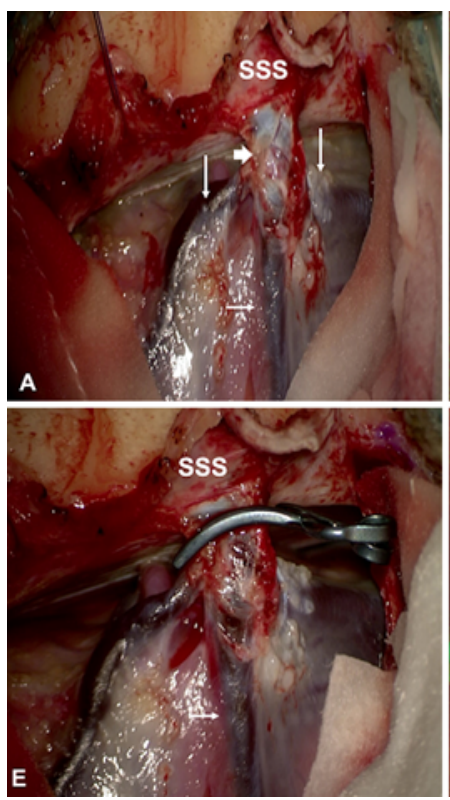
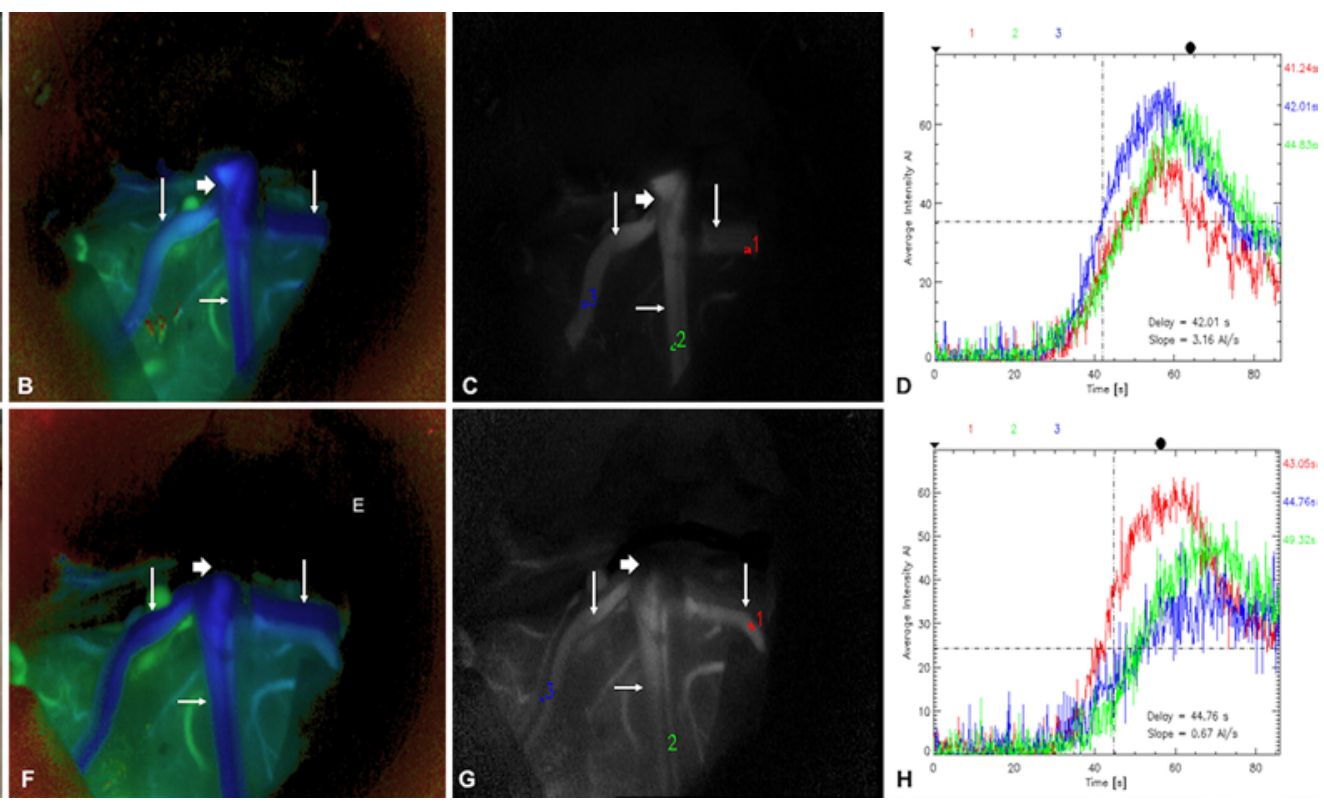

FIG. 7. A: An ICG temporary clipping test of a large parasagittal draining vein consisting of 3 components (thin arrows), with a large collector (thick arrow) connecting to the superior sagittal sinus (SSS), for an interhemispheric approach performed to clip a pericallosal aneurysm. B: The vein exhibited slow flow with slightly different velocities in the 3 tributaries, as shown on the delay map (thinner arrows). C and D: The flow curve analysis confirmed the slight difference in the flow curves. E-H: After clipping of the main collector to the SSS, ICG-VA was performed again and showed a collateral circulation that developed, with the inversion of flow and with the faster component (horizontal thin arrow in $\mathrm{F}, \mathrm{ROI} 1$ in $\mathrm{G}$ and $\mathrm{H}$ ) inversely draining toward the other 2 tributaries (ROIs 2 and 3 in $\mathrm{G}$ and H). This ICG clipping test was considered positive, and the vein was therefore sacrificed without consequences. 
temporary clipping test was negative, showing flow stagnation; for this reason, the vein was not sacrificed. In the 2 slow-draining supracerebellar veins, the ICG temporary clipping test was positive, confirming a collateral retrograde flow after temporary clipping, and the veins could be sacrificed.

In 1 case, the clipping test was performed on a vein located in the posterior median sulcus in the spinal cord (in a case of a spinal ependymoma). In this case, the clipping test, performed directly with bipolar forceps, yielded a positive result, with no flow perturbation during the temporary occlusion, and the vein could be therefore sacrificed.

\section{Discussion}

Our retrospective study showed that ICG-VA with FLOW 800 analysis could be used to intraoperatively evaluate the specific local venous flow patterns in each patient undergoing a neurosurgical procedure. We were able to identify both pathological and physiological patterns of venous flow, based on the disease encountered in the surgical approach. In addition, we used ICG-VA to address the problem of establishing the feasibility of venous sacrifice in different neurosurgical procedures. Even if it is usually considered unsafe to electively sacrifice an important vein, as it could lead to devastating results, $3,20,23,28,35$ venous sacrifice may actually prove to be desirable, under certain circumstances, as it can enlarge the surgical exposure of the targeted site and also increase the extent of resection in tumor surgery. Nonetheless, there are few studies systematically exploring the effect of venous sacrifice in neurosurgical procedures. ${ }^{6,13,14,21,36,37}$ For this reason, most of the time neurosurgeons have based their decision of whether or not to sacrifice a vein on their own surgical experience and on the anatomical location of the vein, rather than on evidence-based data. ${ }^{8}$

Auque and Civit have proposed that the likelihood of producing negative effects after venous sacrifice could generally be predicted by preoperative angiography. ${ }^{2}$ More recently, Nagata and coworkers have also addressed the problem of venous sacrifice by classifying the preoperative angiographic patterns of drainage of those sylvian veins intimately involved in clinoidal meningiomas; they generally recommended avoidance of sacrificing sylvian veins, particularly in the cavernous type of drainage. ${ }^{27}$

ICG-VA, and more recently the evolution of FLOW 800 analysis, has been used to guide neurosurgical procedures, including vascular and tumor disease., $5,7,10,16-18,22,26,29,37$ We were the first group to propose the use of ICG-VA to study the venous flow dynamics and to develop an ICG temporary clipping test to predict the effect of venous sacrifice. ${ }^{13,15}$

In this study, we expanded our experience in venous flow evaluation by applying the FLOW 800 analysis in all cases, with a better interpretation of flow data and understanding the pathophysiology of venous circulation. We identified four patterns of venous flow by ICG-VA: arterialized veins (always related to tumors or vascular malformation), fast-draining veins with anterograde flow, slowdraining veins with anterograde flow, and slow-draining veins with retrograde flow. We also found that the two more common patterns of venous flow (fast-draining draining veins with anterograde flow and slow-draining veins with anterograde flow) could be recognized in different components of the superficial venous system, including veins draining to the superior sagittal, sphenoparietal, and superior petrosal sinuses and the tentorial.

Most of the cases included in this retrospective analysis were operated on with the patient in the supine position; only 4 of the pineal tumors were approached with the patient in a semisitting position. Also, in these 4 cases, we could recognize the presence of fast- and slower-draining veins (supracerebellar veins). Theoretically, there could be a difference in flow characteristics for veins exposed in the posterior fossa when the patient is in the semisitting position, due to the position of the head in relation to the heart and the reduced central venous pressure. Nonetheless, in all patients in whom surgery was performed in the semisitting position, we tried to maintain a positive central venous pressure in order to reduce the risk of venous air embolism; thus, this could be one of the reasons to explain why there was no clear difference in venous flow characteristics. However, the small number of cases studied in the semisitting position does not allow us to generalize this consideration, and this is a consideration that should be analyzed in depth in future studies.

When dealing with arterialized veins, we tried to selectively sacrifice only AVFs directly related to venous arterialization, and we could always check at the end of the procedure to ensure the normalization of venous flow. Furthermore, we found that the specific characteristics of venous flow dynamic of normal veins, as evident on ICG-VA and more precisely on FLOW 800 analysis, were correlated with the potential development of collateral circulation in cases of venous sacrifice. In particular, slow-draining veins with nonuniform fillings, when submitted to a reversible clipping test, presented a prompt flow reversal, confirming the possibility of venous sacrifice in 12 of 13 cases $(92 \%)$. On the other hand, 3 fast-draining veins, when temporarily clipped, always exhibited flow stagnation that was arbitrarily considered to preclude the possibility of their sacrifice. In fact, we assumed that this stagnation was a sign of lack of collateral circulation, possibly associated with an increased risk of postoperative complications related to venous hypertension if the vein were definitely sacrificed. We recognize that this assumption could not necessarily be true in all cases. In fact, even if we could hypothesize the good positive predictive value of the ICG temporary clip testing in the presence of a collateral circulation (we did not have any venous-related complications in this setting when the vein was sacrificed), we could not conduct a similar analysis for the negative predictive value because we did not have cases of fast-draining veins inadvertently sacrificed after a negative ICG clipping test. In addition, even in the 10 cases in which the vein was sacrificed without clipping test (in 7 aneurysms on sylvian bridging veins and in 3 parasagittal meningiomas), we only performed ICG-VA with FLOW 800 analysis at the end of the procedure, always demonstrating a good collateral circulation. We could not exclude the possibility that some of these veins originally had a fast-draining pattern of flow, but un- 
fortunately, we have no data to verify this. Finally, we did not find any case of venous infarction in this retrospective series, and thus we could not establish any correlations between our intraoperative findings and the real occurrence of venous complication. We are fully aware that this could be also associated with a selection bias. In fact, ICG-VA was performed in only around $9 \%$ of the entire population of patients who underwent a neurosurgical procedure for vascular or tumor disease during the same period of time. However, the application of our protocol of venous ICGVA seems to be particularly promising to offer useful and practical intraoperative information regarding individual venous circulation.

\section{Conclusions}

Complete and real-time data on venous drainage patterns and the collateral circulation represent a key point in modern neurosurgery. ICG-VA and FLOW 800 analysis help to identify the patient-specific venous circulation and guide the decision-making process regarding venous sacrifice, with a possible impact on reduction of surgical complications.

\section{References}

1. Acerbi F, Cavallo C, Ferroli P: Intraoperative assessment of blood flow with quantitative indocyanine green videoangiography: the role for diagnosis of regional cerebral hypoperfusion. Neurosurgery 78:E310-E312, 2016 (Letter)

2. Auque J, Civit T: [Superficial veins of the brain.] Neurochirurgie 42 (Suppl 1):88-108, 1996 (Fr)

3. Broggi M, Restelli F, Acerbi F, Ferroli P: Postoperative acute cerebellar swelling after pineal surgery: pathogenesis and treatment. Acta Neurochir (Wien) 158:63-65, 2016

4. Dashti R, Laakso A, Niemelä M, Porras M, Hernesniemi J: Microscope-integrated near-infrared indocyanine green videoangiography during surgery of intracranial aneurysms: the Helsinki experience. Surg Neurol 71:543-550, 2009

5. d'Avella E, Volpin F, Manara R, Scienza R, Della Puppa A: Indocyanine green videoangiography (ICGV)-guided surgery of parasagittal meningiomas occluding the superior sagittal sinus (SSS). Acta Neurochir (Wien) 155:415-420, 2013

6. Davidson L, McComb JG: The safety of the intraoperative sacrifice of the deep cerebral veins. Childs Nerv Syst 29:199-207, 2013

7. de Oliveira JG, Beck J, Seifert V, Teixeira MJ, Raabe A: Assessment of flow in perforating arteries during intracranial aneurysm surgery using intraoperative near-infrared indocyanine green videoangiography. Neurosurgery 62 (6 Suppl 3):1300-1310, 2008

8. Elhammady MS, Heros RC: Cerebral veins: to sacrifice or not to sacrifice, that is the question. World Neurosurg 83:320-324, 2015

9. Endo T, Aizawa-Kohama M, Nagamatsu K, Murakami K, Takahashi A, Tominaga T: Use of microscope-integrated nearinfrared indocyanine green videoangiography in the surgical treatment of intramedullary cavernous malformations: report of 8 cases. J Neurosurg Spine 18:443-449, 2013

10. Ferroli P, Acerbi F, Albanese E, Tringali G, Broggi M, Franzini A, et al: Application of intraoperative indocyanine green angiography for CNS tumors: results on the first 100 cases. Acta Neurochir Suppl 109:251-257, 2011

11. Ferroli P, Acerbi F, Broggi M, Broggi G: Arteriovenous micromalformation of the trigeminal root: intraoperative diagnosis with indocyanine green videoangiography: case report. Neurosurgery 67 (3 Suppl Operative):E309-E310, 2010
12. Ferroli P, Acerbi F, Broggi M, Broggi G: The role of indocyanine green videoangiography (ICGV) in surgery of parasagittal meningiomas. Acta Neurochir (Wien) 155:1035, 2013

13. Ferroli P, Acerbi F, Tringali G, Albanese E, Broggi M, Franzini A, et al: Venous sacrifice in neurosurgery: new insights from venous indocyanine green videoangiography. J Neurosurg 115:18-23, 2011

14. Ferroli P, Nakaji P, Acerbi F, Albanese E, Broggi G: Indocyanine green (ICG) temporary clipping test to assess collateral circulation before venous sacrifice. World Neurosurg 75:122-125, 2011

15. Ferroli P, Tringali G, Albanese E, Broggi G: Developmental venous anomaly of petrous veins: intraoperative findings and indocyanine green video angiographic study. Neurosurgery 62:ONS418-ONS421, 2008

16. Hao S, Li D, Ma G, Yang J, Wang G: Application of intraoperative indocyanine green videoangiography for resection of spinal cord hemangioblastoma: advantages and limitations. J Clin Neurosci 20:1269-1275, 2013

17. Hojo M, Arakawa Y, Funaki T, Yoshida K, Kikuchi T, Takagi Y, et al: Usefulness of tumor blood flow imaging by intraoperative indocyanine green videoangiography in hemangioblastoma surgery. World Neurosurg 82:e495-e501, 2014

18. Hope-Ross M, Yannuzzi LA, Gragoudas ES, Guyer DR, Slakter JS, Sorenson JA, et al: Adverse reactions due to indocyanine green. Ophthalmology 101:529-533, 1994

19. Jang WY, Jung S, Jung TY, Moon KS, Kim IY: Predictive factors related to symptomatic venous infarction after meningioma surgery. Br J Neurosurg 26:705-709, 2012

20. Kiya K, Satoh H, Mizoue T, Kinoshita Y: Postoperative cortical venous infarction in tumours firmly adherent to the cortex. J Clin Neurosci 8 (Suppl 1):109-113, 2001

21. Liebelt BD, Barber SM, Desai VR, Harper R, Zhang J, Parrish $\mathrm{R}$, et al: Superior petrosal vein sacrifice during microvascular decompression: perioperative complication rates and comparison with venous preservation. World Neurosurg 104:788-794, 2017

22. Maduri R, Guyotat J, Signorelli F: Semi-quantitative assessment of flow dynamics during indocyanine green video-angiography in the treatment of intracranial dural arteriovenous fistulas: how I do it. Acta Neurochir (Wien) 158:1387-1391, 2016

23. Masuoka J, Matsushima T, Hikita T, Inoue E: Cerebellar swelling after sacrifice of the superior petrosal vein during microvascular decompression for trigeminal neuralgia. J Clin Neurosci 16:1342-1344, 2009

24. Mathiesen T, Pettersson-Segerlind J, Kihlström L, Ulfarsson E: Meningiomas engaging major venous sinuses. World Neurosurg 81:116-124, 2014

25. McNatt SA, Sosa IJ, Krieger MD, McComb JG: Incidence of venous infarction after sacrificing middle-third superior sagittal sinus cortical bridging veins in a pediatric population. J Neurosurg Pediatr 7:224-228, 2011

26. Motoyama Y, Gurung P, Takeshima Y, Nakagawa I, Park YS, Nakase H: Indocyanine green (ICG) videoangiographyguided dissection of the sylvian fissure on the transsylvian approach: technical note. World Neurosurg 87:45-47, 2016

27. Nagata T, Ishibashi K, Metwally H, Morisako H, Chokyu I, Ichinose T, et al: Analysis of venous drainage from sylvian veins in clinoidal meningiomas. World Neurosurg 79:116123,2013

28. Nakase H, Shin Y, Nakagawa I, Kimura R, Sakaki T: Clinical features of postoperative cerebral venous infarction. Acta Neurochir (Wien) 147:621-626, 2005

29. Nussbaum ES, Defillo A, Nussbaum L: The use of indocyanine green videoangiography to optimize the dural opening for intracranial parasagittal lesions. Neurosurgery 70 (1 Suppl Operative):61-64, 2012

30. Raza SM, Gallia GL, Brem H, Weingart JD, Long DM, Olivi 
A: Perioperative and long-term outcomes from the management of parasagittal meningiomas invading the superior sagittal sinus. Neurosurgery 67:885-893, 2010

31. Schaller B, Graf R: Cerebral venous infarction: the pathophysiological concept. Cerebrovasc Dis 18:179-188, 2004

32. Schmid-Elsaesser R, Steiger HJ, Yousry T, Seelos KC, Reulen HJ: Radical resection of meningiomas and arteriovenous fistulas involving critical dural sinus segments: experience with intraoperative sinus pressure monitoring and elective sinus reconstruction in 10 patients. Neurosurgery 41:10051018,1997

33. Sindou M, Auque J, Jouanneau E: Neurosurgery and the intracranial venous system. Acta Neurochir Suppl 94:167175,2005

34. Sughrue ME, Rutkowski MJ, Shangari G, Fang S, Parsa AT, Berger MS, et al: Incidence, risk factors, and outcome of venous infarction after meningioma surgery in 705 patients. J Clin Neurosci 18:628-632, 2011

35. Suzuki Y, Endo T, Ikeda H, Ikeda Y, Matsumoto K: Venous infarction resulting from sacrifice of a bridging vein during clipping of a cerebral aneurysm: preoperative evaluation using three-dimensional computed tomography angiographycase report. Neurol Med Chir (Tokyo) 43:550-554, 2003

36. Takagi Y, Sawamura K, Hashimoto N, Miyamoto S: Evaluation of serial intraoperative surgical microscope-integrated intraoperative near-infrared indocyanine green videoangiography in patients with cerebral arteriovenous malformations. Neurosurgery 70 (1 Suppl Operative):34-43, 2012

37. Ye X, Liu XJ, Ma L, Liu LT, Wang WL, Wang S, et al: Clinical values of intraoperative indocyanine green fluorescence video angiography with Flow 800 software in cerebrovascular surgery. Chin Med J (Engl) 126:4232-4237, 2013

\section{Disclosures}

Dr. Acerbi received fees from Carl Zeiss Meditec for lectures at international meetings.

\section{Author Contributions}

Conception and design: Acerbi, Vetrano, Ferroli. Acquisition of data: Acerbi, Vetrano, Sattin, Falco, de Laurentis, Zattra. Analysis and interpretation of data: Acerbi, Vetrano, Sattin, Falco, Zattra, Bosio, Rossini, Schiariti, Ferroli. Drafting the article: Acerbi, Vetrano, Sattin, Falco, Bosio, Broggi, Schiariti. Critically revising the article: Acerbi, Vetrano, Sattin, Falco, Rossini, Broggi, Schiariti, Ferroli. Reviewed submitted version of manuscript: Acerbi, Vetrano, Sattin, Falco, de Laurentis, Zattra, Rossini, Broggi, Ferroli. Approved the final version of the manuscript on behalf of all authors: Acerbi. Study supervision: Acerbi, Ferroli.

\section{Supplemental Information Videos}

Video 1. https://vimeo.com/268379922.

Video 2. https://vimeo.com/268379944.

Video Abstract. https://vimeo.com/273358370.

\section{Correspondence}

Francesco Acerbi: Fondazione IRCCS "Istituto Neurologico C. Besta," Milan, Italy. francesco.acerbi@istituto-besta.it. 